\title{
Carcinoma escamocelular del canal anal metastásico en un paciente con VIH tratado con quimiorradioterapia concomitante. Reporte de un caso y revisión de la literatura
}

\section{Metastatic anal canal squamous cell carcinoma in a patient with HIV treated with concomitant radiotherapy chemo. Case report and literature review}

\author{
Handerson Rafael Osma Ch. ${ }^{*}$, Carlos Eduardo Bonilla G. ${ }^{2}$, Ricardo Oliveros W. ${ }^{3}$, Henry Alexander Vargas D. ${ }^{4}$, Julián Alberto Beltrán S. ${ }^{5}$, \\ Mario Alexander Melo U. ${ }^{6}$
}

Especialista en Medicina Interna, Universidad Nacional de Colombia, Instituto Nacional de Cancerología. Bogotá, Colombia.

2 Médico especialista en Medicina Interna y Oncología, especialista en Docencia Universitaria, Servicio de Oncología Clínica en el Instituto Naciona de Cancerología. Bogotá, Colombia.

${ }^{3}$ Médico especialista en Cirugía General y Cirugía Gastrointestinal. Coordinador del Servicio de Gastroenterología del Instituto Nacional de Cancerología. Bogotá, Colombia.

${ }^{4}$ Médico especialista en Medicina Interna. Fellow de Oncología, Servicio de Oncología Clínica Instituto Nacional de Cancerología. Bogotá, Colombia.

${ }_{5}$ Médico especialista en Radiología, Neurorradiología y Neurorradiología Intervencionista. Servicio de Radiología del Instituto Nacional de Cancerología. Bogotá, Colombia.

${ }^{6}$ Médico especialista en Patología. Servicio de Patología del Instituto Nacional de Cancerología. Bogotá, Colombia.

*Correspondencia: Handerson Rafael Osma, hrosmac@unal.edu.co

Fecha recibido: $\quad 26 / 08 / 18$ Fecha aceptado: 01/11/18

\begin{abstract}
Resumen
El carcinoma del canal anal es responsable de hasta el $4 \%$ de los casos de cáncer de colon, recto y ano; el tipo histológico más común es el carcinoma escamocelular. Una proporción no despreciable de pacientes se presenta con enfermedad metastásica al momento del diagnóstico. En estos estadios, el pronóstico es pobre y su tratamiento usualmente se basa en quimioterapia paliativa con cisplatino y 5 -fluorouracilo, con tasas de supervivencia que no superan el $30 \%$ a 5 años. Algunos estudios recientes han sugerido que el tratamiento multidisciplinario con quimiorradiación, que se brinda en estadios más tempranos de la enfermedad, podría mejorar la supervivencia en un grupo seleccionado de pacientes.

A continuación, presentamos el caso de un paciente masculino de 54 años con carcinoma escamocelular del canal anal con compromiso metastásico extenso y con VIH atendido en una institución especializada en el manejo de cáncer, en el cual se logró remisión completa de la enfermedad luego del manejo con quimiorradioterapia concomitante con mitomicina C y 5 -fluorouracilo y se mantiene en remisión después de cerca de 4 años de haberse suspendido el tratamiento. Se discute el caso y se revisa la literatura al respecto.
\end{abstract}

Palabras clave

Canal anal, neoplasias del ano, carcinoma de células escamosas, VIH, quimiorradioterapia.

\begin{abstract}
Anal canal carcinoma is responsible for up to $4 \%$ of all cases of colon, rectum and anus cancer. The most common histological type is squamous cell carcinoma. A non-negligible proportion of patients have metastasized by the time of diagnosis. In these stages the prognosis is poor, and treatment is usually based on palliative chemotherapy with cisplatin and 5 -fluorouracil. Five year survival rates do not exceed $30 \%$. Some recent studies have suggested that multidisciplinary chemoradiotherapy (chemotherapy combined with radiation therapy) in earlier stages of the disease could improve survival for a select group of patients.

We present the case of a 54-year-old male patient with squamous cell carcinoma of the anal canal with extensive metastasis who also had HIV. He was treated at an institution specializing in cancer treatment where complete remission of the disease was achieved after treatment with chemoradiotherapy with Mitomycin $\mathrm{C}$ and 5 -fluorouracil. He remains in remission four years after discontinuation of treatment. We discuss the case and review the literature.
\end{abstract}

Keywords

Anal canal, neoplasms of the anus, squamous cell carcinoma, HIV, chemoradiotherapy. 


\section{INTRODUCCIÓN}

El carcinoma de canal anal representa el $2 \%$ a $4 \%$ de todos los casos de cáncer de colon, recto y ano (1). Su pico de presentación ocurre entre los 58 y 64 años y su frecuencia ha venido en aumento. En Estados Unidos, las tasas de incidencia han pasado de 0,8 casos por 100000 habitantes por año en 1975 a 1,5 casos por 100000 habitantes por año en el 2011, posiblemente por el impacto de la infección por virus de inmunodeficiencia humana (VIH) (2). El tipo histológico más común es el carcinoma de células escamosas, que representa $85 \%$ a $90 \%$ de todos los casos $(2,3)$. La mitad de los pacientes tiene enfermedad localizada al momento del diagnóstico, una tercera parte se presentan como enfermedad regional nodal y $10 \%-15 \%$ tiene metástasis a distancia (4).

Hay poca información con respecto a la historia natural del carcinoma escamocelular metastásico, debido especialmente a la escasez de estudios prospectivos en esta patología (5). En estados avanzados, la National Comprehensive Cancer Network (NCCN) recomienda la quimioterapia sistémica paliativa basada en cisplatino y 5-fluorouracilo $y$, en algunos pacientes, la radioterapia paliativa de forma secuencial (no concomitante) (6). En estos casos, la quimioterapia ofrece una tasa de respuesta razonable, pero pobres desenlaces fuertes, con una probabilidad de supervivencia a 5 años que va entre el 15\% y 30\% (7). Evidencia reciente sugiere que en algunos pacientes con enfermedad metastásica bien seleccionados, el tratamiento multidisciplinario con quimiorradiación concomitante podría mejorar los desenlaces de supervivencia (8).

A continuación, presentamos el caso de un paciente con carcinoma escamocelular del canal anal metastásico con VIH atendido en una institución especializada en el manejo de cáncer, en quien se brindó tratamiento con quimiorradioterapia concomitante con 5-fluorouracilo y mitomicina C y luego quimioterapia sola con el mismo protocolo por 6 ciclos, lo que logró una respuesta completa sostenida en el tiempo, persistiendo en remisión después de casi 4 años de haber suspendido el tratamiento. Se discute el caso y se revisa la literatura al respecto.

\section{DESCRIPCIÓN DEL CASO}

Paciente masculino de 54 años, que consulta en mayo de 2013 por dolor y sensación de masa en región recto-anal. Tenía antecedente de infección por VIH, en manejo con terapia HAART desde diciembre de 2012, con recuento de linfocitos CD4 de 57 células/ $\mu \mathrm{L}$ y carga viral 40612 copias $/ \mathrm{mL}(\log 5.6)$. Al examen físico se encontró un paciente con sarcopenia y múltiples adenopatías en zona IV izquierda del cuello, axilares e inguinales. A la explo- ración de la región perineal se encontraron hemorroides externas edematizadas, al menos 5 nódulos subcutáneos en región glútea de hasta $5 \mathrm{~mm}$ de diámetro, y al tacto rectal una lesión que iniciaba por encima del reborde anal sobre la cara anterior del canal anal y recto distal. El resto del examen físico estaba dentro de los parámetros normales.

Le realizaron una rectosigmoidoscopia, en donde se evidenció una lesión neoplásica en la ampolla rectal a $5 \mathrm{~cm}$ del reborde anal, cuya biopsia reportó un carcinoma escamocelular moderadamente diferenciado. Una resonancia magnética de pelvis evidenció, a nivel del canal anal, un engrosamiento neoplásico con zona de ulceración focal anterior en contacto con la uretra membranosa y ápex de la próstata. La resonancia magnética de abdomen reportó lesiones metastásicas en glándula suprarrenal derecha, adenopatías paraaórticas izquierdas e infrarrenales, retrocavas, en eje celíaco y retrocrurales derechas. La tomografía de tórax reportó múltiples adenopatías tumorales en región axilar derecha, supraclaviculares, prevasculares, paraesofágicas, retrocrurales derechas y paraaórticas izquierdas. Adicionalmente mostró una lesión nodular cavitada en la base del pulmón izquierdo y múltiples nódulos subcentimétricos con densidad de tejidos blandos de distribución aleatoria, sospechosos de malignidad (Figura 1).

Se realizó biopsia excisional de un ganglio axilar derecho para descartar otro primario, el cual confirma compromiso metastásico a distancia por carcinoma escamocelular del canal anal, con desdiferenciación sarcomatosa. Se hizo el diagnóstico de carcinoma escamocelular del canal anal en estadio IV por T4N2M1 (American Joint Committee on Cancer, versión 7) (9). A pesar de la extensa enfermedad a distancia, teniendo en cuenta el buen estado general del paciente y con miras a brindarle la máxima respuesta posible, se decidió brindar el tratamiento que se reserva para estadios más tempranos: quimiorradioterapia concomitante con mitomicina C $10 \mathrm{mg} / \mathrm{m}^{2}$ días 1 y 29 y 5-fluorouracilo $1000 \mathrm{mg} / \mathrm{m}^{2} /$ día en infusión continua los días 1 a 4 y 29 a 32 de la radioterapia. Recibió este tratamiento entre el 20 de agosto y el 05 de octubre de 2013, sin toxicidad limitante. Se obtuvo respuesta clínica completa en canal anal y por tomografías una respuesta parcial dada por disminución mayor del $30 \%$ del conglomerado ganglionar en la estación IV izquierda y axilar derecha, resolución del componente ganglionar mediastinal, disminución del tamaño de las lesiones pulmonares, resolución de algunas adenopatías abdominales y disminución del tamaño de las lesiones de la glándula suprarrenal derecha (Figura 2).

Ante la buena respuesta obtenida se decidió continuar tratamiento sistémico con el mismo régimen de quimioterapia de mitomicina $\mathrm{C}+5$-fluorouracilo, con monitorización hematológica y renal estrecha. El paciente completó 6 ciclos el 10 de octubre de 2014 y desde entonces se dejó en 

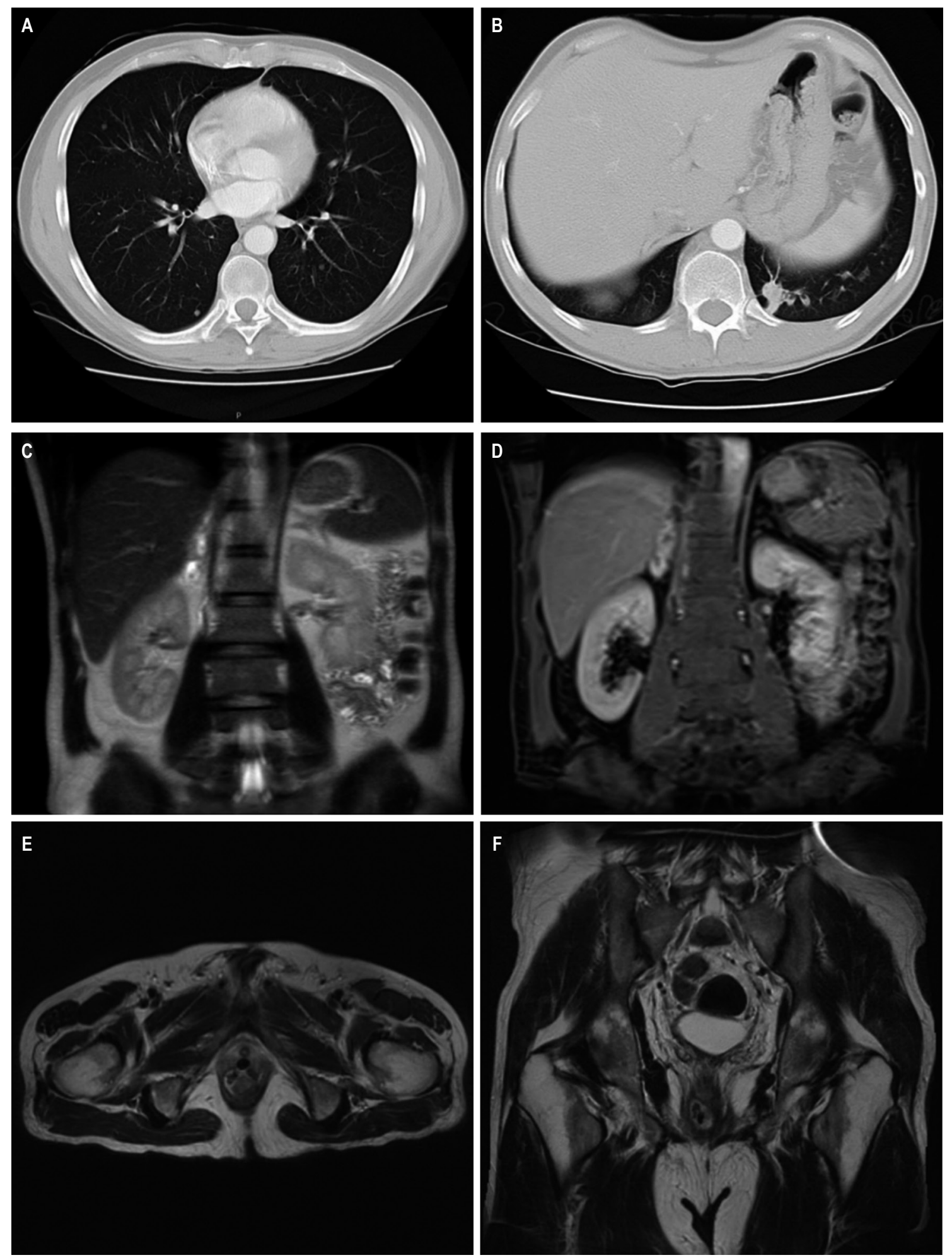

Figura 1. Imágenes del paciente al momento del diagnóstico. A y B. TAC de tórax. Proyección axial. Múltiples nódulos subcentimétricos con densidad de tejidos blandos de distribución aleatoria y una lesión nodular dominante en la base pulmonar izquierda. C y D. RM de abdomen. Proyección coronal. Se evidencian dos lesiones focales en la glándula suprarrenal derecha: una de ellas en el brazo medial de $14 x 17 \mathrm{~mm}$ y otra en el cuerpo de la glándula de $14 x$ $19 \mathrm{~mm}$, las cuales son hiperintensas en T2 (C) e hipointensas en T1 (D), no suprimen señal en secuencias fuera de fase, presentan restricción periférica a la difusión y realce periférico al medio de contraste en relación con metástasis necróticas (estas dos últimas secuencias no ilustradas). E y F. RM de pelvis. Proyecciones axial y coronal en secuencia T2. Se evidencia engrosamiento concéntrico y de aspecto neoplásico con zona de ulceración anterior en el canal anal, con espesor de $14 \mathrm{~mm}$ y presencia de realce moderado con el medio de contraste en contacto con la uretra membranosa, sin fístulas a este órgano. 

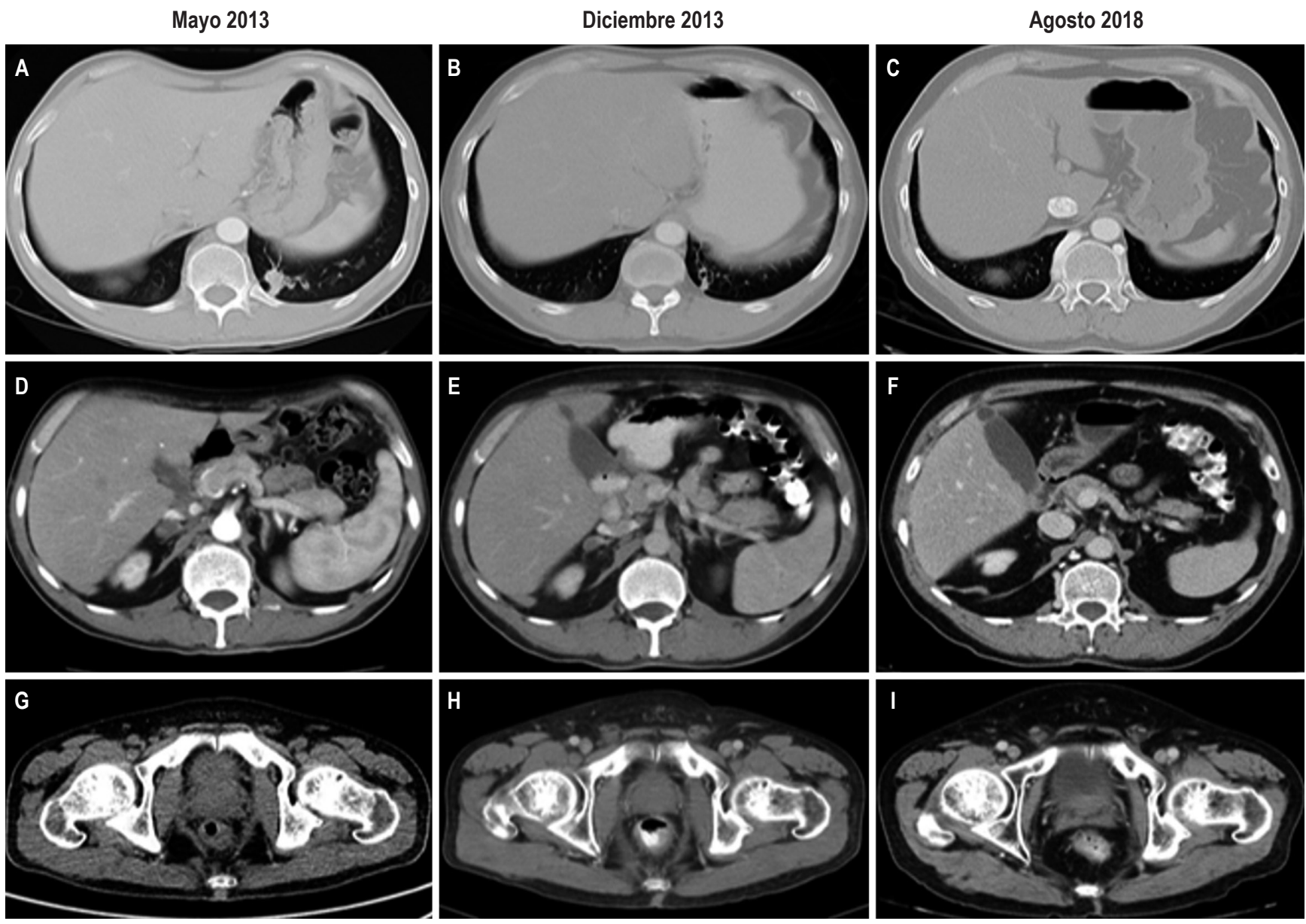

Figura 2. Seguimiento tomográfico del paciente. A-C. Las imágenes muestran la evolución del tamaño del nódulo metastásico en la base del pulmón izquierdo, con resolución completa a fecha del último seguimiento. D-F. Se evidencia la evolución de una de las lesiones metastásicas en la glándula suprarrenal derecha, con respuesta completa en el último estudio. G-I. Las imágenes muestran la respuesta parcial a nivel del canal anal, con enfermedad estable a agosto de 2018 .

seguimiento. Las tomografías de reevaluación de fin de tratamiento mostraron respuesta pulmonar, ganglionar axilar y cervical completa, persistiendo solo algunas adenomegalias retrocrurales y retroperitoneales izquierdas residuales y estabilidad de las lesiones en la glándula suprarrenal derecha. Desde entonces ha continuado en seguimiento clínico e imagenológico y hasta el control de agosto de 2018 permanece con dichas lesiones estables, sin evidencia de progresión de la enfermedad (Figura 2).

\section{DISCUSIÓN}

La mayoría de la literatura sobre el tratamiento del carcinoma escamocelular de canal anal metastásico se limita a reportes y series de casos en los que se han extrapolado los protocolos de quimioterapia usados para carcinomas escamocelulares de otras localizaciones, como pulmón y cérvix (10). El paradigma oncológico considera que, en este estadio, la enfermedad no es curable, por lo que para su tratamiento, las guías más importantes de oncología a nivel mundial aconsejan el uso de poliquimioterapia con intención paliativa y se deja como opcional el uso de radioterapia pélvica (secuencial, no concomitante) en dosis paliativas en caso de enfermedad primaria voluminosa y sintomática $(1,6)$. En estadios metastásicos se han reportado medianas de supervivencia libre de progresión de 5 a 7 meses y de supervivencia global entre 15 y 22 meses $(7,8)$.

El tratamiento con base en fluorinados más platinos ha reportado tasas de respuesta global en casi $60 \%$ de los pacientes, casi todas respuestas parciales, con medianas de supervivencia de hasta 34,5 meses, pero sin respuestas sostenidas a largo plazo $(11,12)$. Una serie retrospectiva en el MD Anderson demostró una mediana de supervivencia libre de progresión de 7,2 meses y de supervivencia global 
de 38 meses (13). Otros esquemas utilizados incluyen la combinación de carboplatino/paclitaxel, con una mediana de supervivencia global de 12 meses (14). La combinación de paclitaxel, carboplatino y 5-FU se evaluó en un estudio fase II, que probó la eficacia de esta terapia en el tratamiento de carcinoma escamocelular de varios sitios primarios, en el que se demostró para los 7 pacientes de sitio primario ano una respuesta global en 4 de ellos, pero con una considerable tasa de efectos secundarios, que incluyó leucopenia (48\%), mucositis (28\%) y diarrea (17\%) (15). En 2006, Jhawer y colaboradores estudiaron el uso de mitomicina $\mathrm{C}$, adriamicina, cisplatino y bleomicina, CCNU, y obtuvieron una tasa de respuesta global del $60 \%$, una mediana de supervivencia libre de progresión de 8 meses y de supervivencia global de 15 meses, a costa de una toxicidad severa que incluyó leucopenia y trombocitopenia (10\%), vómito (10\%), calambres (5\%) y arritmia cardíaca (5\%) (16).

Como ya se expuso, el pronóstico de pacientes con enfermedad metastásica es muy pobre. El paciente de nuestro caso logró respuesta completa a nivel local y respuesta sistémica parcial con la quimiorradioterapia concomitante inicial (esquema estándar en enfermedad no metastásica) y, después de 6 ciclos de mitomicina $\mathrm{C}+5$-fluorouracilo, el paciente logró respuesta completa, que se ha mantenido durante cerca de 4 años. La quimiorradioterapia secuencial en enfermedad metastásica está recomendada en las guías de manejo internacional, bajo recomendación de expertos; no obstante, a pesar de que existe escasa evidencia acerca del impacto que pueda tener este tipo de tratamiento y el momento óptimo para su inicio, pequeñas series de caso han mostrado mejorar la supervivencia libre de enfermedad a largo plazo (17). La duración óptima de la terapia para quienes logran una respuesta adecuada tampoco ha sido establecida, algunos autores sugieren continuar el tratamiento de manera indefinida para lograr una máxima respuesta si se tolera adecuadamente (10).

En el escenario metastásico, las guías sugieren utilizar la radioterapia solo si se usa en combinación con 5-FU y un platino. Aquí, exponemos una experiencia exitosa utilizando mitomicina $C$ en lugar de cisplatino, en la que se logró una respuesta casi completa y estabilidad de las lesiones luego de 4 años de haber finalizado el esquema Nigro. Actualmente se encuentra en curso un estudio fase II que compara el uso de cisplatino $+5 \mathrm{FU} v$ s. carboplatino con paclitaxel semanal para pacientes con carcinoma escamocelular de canal anal metastásico o localmente recurrente e inoperable. El objetivo principal del estudio es evaluar la tasa de respuesta y los desenlaces secundarios incluyen supervivencia libre de progresión, supervivencia global, toxicidad y calidad de vida (18).

Los pacientes con VIH con carcinoma de canal anal tienen un peor pronóstico comparados con pacientes sero- negativos y pueden experimentar una mayor toxicidad con quimioterapia, especialmente cuando el recuento de CD4 es $<200$ células/ $\mu \mathrm{L}$, pudiendo requerir períodos de descanso más prolongados y reducción en la dosis de los agentes quimioterapéuticos $(19,20)$. Las principales toxicidades que experimentan han sido hematológicas, gastrointestinales y radiodermitis. También pueden requerir reducción del campo de radiación, utilizando radioterapia con intensidad modulada (IMRT) (21). Si bien algunos autores han encontrado una tendencia en mejoría de la supervivencia con recuentos de CD4 $>200$, la evidencia no es contundente y el impacto del sistema inmune de los pacientes con VIH y carcinoma escamocelular de canal anal y su terapia HAART asociada sigue estando por establecer $(21,22)$. Adicionalmente, los pacientes con VIH han sido excluidos de grandes estudios de carcinoma escamocelular de canal anal, como el ACT I, RTOG 98-11 y ACCORD 03 (23). En nuestro caso, el paciente tuvo una tolerancia aceptable del tratamiento con quimiorradioterapia y la terapia antirretroviral concomitante, no hubo requerimientos de períodos de descanso prolongados y se cumplieron todos los ciclos de quimioterapia.

En los últimos años ha cobrado importancia el uso de la terapia dirigida para tratamiento de cáncer, como es el caso de los trabajos de los doctores Allison Y Honjo en inmunoterapia contra el cáncer, con los cuales fueron galardonados con el premio Nobel de Fisiología o Medicina de 2018 (24, 25). El rol que tiene el virus de papiloma humano (VPH) en la carcinogénesis del carcinoma escamocelular de canal anal, sobre todo en pacientes con inmunosupresión, ha permitido el uso de anticuerpos antiPD-1. Dos estudios recientes han estudiado el uso de estas moléculas. En 2017, Ott y colaboradores estudiaron los resultados de seguridad y eficacia del pembrolizumab en la cohorte de pacientes con carcinoma localmente avanzado o metastásico del canal anal PDL-1 positivos del estudio fase I KEYNOTE-028, con falla en al menos una terapia estándar previa. Los desenlaces primarios fueron la seguridad y la tasa de respuesta global y como desenlaces secundarios la supervivencia global, libre de progresión y duración de la respuesta. 25 pacientes con una mediana de edad de 63 años fueron aleatorizados, de los cuales el $64 \%$ experimentó eventos adversos relacionados con el tratamiento, sin que hubiera ninguna muerte relacionada con este. 4 de ellos tuvieron una respuesta parcial, la tasa de supervivencia global fue de $17 \%$ (IC $95 \% 5 \%-37 \%$ ) y el $42 \%$ tuvo enfermedad estable durante una mediana de 3,6 meses. La mediana de supervivencia libre de progresión fue de 3 meses y la de supervivencia global fue de 9,3 meses. Los autores concluyeron que esta molécula demostró un perfil de seguridad manejable y aceptable actividad antitumoral (26). 
En otro estudio fase II, realizado por Morris y colaboradores, se valoró la eficacia de nivolumab en pacientes con carcinoma escamocelular de canal anal metastásico que no habían recibido previamente inmunoterapia. Se aleatorizaron un total de 39 pacientes, con una mediana de edad de 56 años. 7 pacientes (21\%) tuvieron una respuesta parcial y poco más de la mitad alcanzó enfermedad estable, con una tasa de control de la enfermedad del $79 \%$. La mediana de supervivencia libre de progresión fue de 4,1 meses y de supervivencia global de 11,5 meses y los efectos adversos más comunes fueron fatiga, náusea y sarpullido. Los autores concluyeron que nivolumab demostró una actividad potencialmente significativa, con una adecuada tolerancia (27).

También se ha estudiado los inhibidores del factor de crecimiento epidérmico (EGFR). El uso de cetuximab se ha descrito en algunos reportes de caso de pacientes con KRAS wild-type y en análisis retrospectivos se encontró inicialmente un posible rol de este medicamento para pacientes con carcinoma escamocelular de canal anal (28). Sin embargo, es necesario tener en cuenta los desenlaces de seguridad encontrados cuando se ha utilizado los inhibidores del EGFR en pacientes con enfermedad localmente avanzada en combinación con quimiorradioterapia, en donde se han encontrado tasas de toxicidad grado $3 / 4$ de hasta el $90 \%$ y con tasas no muy buenas de respuesta, que ha llevado a recomendar continuar en la búsqueda de alternativas terapéuticas más eficaces y menos tóxicas, por lo que los desenlaces de eficacia y seguridad en el escenario metastásico aún deben comprobarse en ensayos clínicos aleatorizados y estudios prospectivos (29).

\section{CONCLUSIONES}

Este caso ilustrativo nos permite considerar que en pacientes seleccionados con enfermedad metastásica distante es posible brindar el esquema de quimiorradioterapia concomitante en dosis curativas, que se usa de forma estándar en enfermedad localizada, con lo cual se podría lograr respuesta completa y sostenida a nivel locorregional y control de la enfermedad sistémica. La serie de casos del MD Anderson apoya también este abordaje, lo cual puede significar el punto inicial para realizar un cambio en el paradigma oncológico en cuanto al pronóstico y tratamiento de esta enfermedad. Se requieren ensayos clínicos aleatorizados con seguimiento de los pacientes a largo plazo que permitan confirmar esta hipótesis y tener evidencia de mejor calidad con respecto al tratamiento del carcinoma escamocelular de canal anal en estadio IV.

\section{Fuente de financiación}

Recursos propios.

\section{Agradecimientos}

A todos los pacientes del Instituto Nacional de Cancerología, por ser nuestro motivo de la incesante lucha contra el cáncer.

\section{REFERENCIAS}

1. Glynne-Jones R, Nilsson P, Aschele C, Goh V, Peiffert D, Cervantes A, et al. Anal cancer: ESMO-ESSO-ESTRO clinical practice guidelines for diagnosis, treatment and followup. Eur J Surg Oncol. 2014;40:1165-76. doi: https://doi. org/10.1016/j.ejso.2014.07.030.

2. Nelson R, Levine A, Bernstein L, Smith D, Lai L. Changing patterns of anal canal carcinoma in the United States. J Clin Oncol. 2013;31:1569-75. doi: https://doi.org/10.1200/ JCO.2012.45.2524.

3. Oliveros R, Rey-León C, Olarte H, Ospina J, Villamizar J, Acosta M, et al. Carcinoma del ano: experiencia con 82 pacientes. Rev Col Cir. 1990;5(3):139-46.

4. Abbas A, Yang G, Fakih M. Management of anal cancer in 2010. Part 1: Overview, screening, and diagnosis. Oncology (Williston Park). 2010;24:364-9.

5. Glynne-Jones R. Chemotherapy, "Lighting the fire" or releasing the brakes" in metastatic anal cancer. Dis Colon Rectum. 2017;60(8):757-8. doi: https://doi.org/10.1097/ DCR.0000000000000828.

6. National Comprehensive Cancer Network. Anal carcinoma. V.1.2018. Último acceso:24 de mayo de 2018. Disponible en: https://www.nccn.org/professionals/physician_gls/ pdf/anal.pdf (nota del corrector: el link no lleva al documento, revisar).

7. Sclafani F, Morano F, Cunningham D, Baratelli C, Kalaitzaki E, Watkins D, et al. Platinum-fluoropyrimidine and paclitaxel-based chemotherapy in the treatment of advanced anal cancer patients. Oncologist. 2017;22(4):402-8. doi: https://doi.org/10.1634/theoncologist.2016-0241.

8. Eng C, Chang G, You Y, Das P, Bigas M, Xing Y, et al. The role of systemic chemotherapy and multidisciplinary management in improving the overall survival of patients with metastatic squamous cell carcinoma of the anal canal. Oncotarget. 2014;5:11133-42. doi: https://doi. org/10.18632/oncotarget.2563.

9. Edge S, Byrd D, Compton C, Fritz A, Greene F, Trotti A. AJCC cancer staging manual. New York, NY: Springer. 2009.

10. Shridhar R, Shibata D, Chan E, Thomas C. Anal cancer: current standards in care and recent changes in practice. CA Cancer J Clin. 2015;65(2):139-62. doi: https://doi. org/10.3322/caac.21259.

11. Eng C, Pathak P. Treatment options in metastatic squamous cell carcinoma of the anal canal. Curr Treat Options Oncol. 2008;9(4-6):400-7. doi: https://doi.org/10.1007/s11864009-0103-7.

12. Faivre C, Rougier P, Ducreux M, Mitry E, Lusinchi A, Lasser $\mathrm{P}$, et al. 5-fluorouracil and cisplatin combination chemothe- 
rapy for metastatic squamous-cell anal cancer. Bull Cancer. 1999;86(10):861-5.

13. Pathak P, King BT, Ohinata A, Das P, Crane CH, Chase $\mathrm{JL}$, et al. The treatment of metastatic squamous cell carcinoma (SCCA) of the anal canal: a single institution experience. In ASCO Gastrointestinal Cancers Symposium: Multidisciplinary Approaches to the Prevention, Diagnosis, and Therapy of GI Cancers. 2008.

14. Kim R, Byer J, Fulp W, Mahipal A, Dinwoodie W, Shibata D. Carboplatin and paclitaxel treatment is effective in advanced anal cancer. Oncology. 2014;87:125-32. doi: https://doi. org/10.1159/000361051.

15. Hainsworth J, Burris H 3rd, Meluch A, Baker M, Morrissey L, Greco F. Paclitaxel, carboplatin, and long-term continuous infusion of 5-fluorouracil in the treatment of advanced squamous and other selected carcinomas: results of a Phase II trial. Cancer. 2001;92(3):642-9. doi: https:// doi.org/10.1002/1097-0142(20010801)92:3<642::AIDCNCR1365>3.0.CO;2-Z.

16. Jhawer M, Mani S, Lefkopoulou M, Hanh R, Harris J, Catalano $\mathrm{P}$, et al. Phase II study of mitomycin-C, adriamycin, cisplatin (MAP) and bleomycin-CCNU in patients with advanced cancer of the anal canal: an eastern cooperative oncology group study E7282. Investig New Drugs. 2006;24(5):447-54. doi: https://doi.org/10.1007/s10637006-7667-x.

17. Rogers J, Crane C, Das P, Delclos M, Gould S, Ohinata A, et al. Definitive chemoradiation in oligometastatic squamous cell carcinoma of the anal canal. Gastrointest Cancer Res. 2014;7(2):65-8.

18. Sclafani F, Adams R, Eng C, Benson A, Glynne-Jones R, Sebag-Montefiore D, et al. Inter AACT: an international multicenter open label randomized phase II advanced anal cancer trial comparing cisplatin (CDDP) plus 5-fluorouracil (5-FU) versus carboplatin (CBDCA) plus weekly paclitaxel (PTX) in patients with inoperable locally recurrent (ILR) or metastatic disease. J Clin Oncol. 2015;33(3):TPS792. doi: https://doi.org/10.1200/jco.2015.33.3_suppl.tps792.

19. Bryant A, Huynh-Le M, Simpson D, Gupta S, Sharabi A, Murphy J. Association of HIV status with outcomes of anal squamous cell carcinoma in the era of highly active antiretroviral therapy. JAMA Oncol. 2018;4(1):120-2. doi: https://doi.org/10.1001/jamaoncol.2017.2844.

20. Hoffman R, Welton M, Klencke B, Weinberg V, Krieg R. The significance of pretreatment CD4 count on the outcome and treatment tolerance of HIV-positive patients with anal cancer. Int J Radiat Oncol Biol Phys. 1999;44(1):127-31. doi: https://doi.org/10.1016/S0360-3016(98)00528-8.

21. Kauh J, Koshy M, Gunthel C, Joyner M, Landry J, Thomas C Jr. Management of anal cancer in the HIV-positive population. Oncology (Williston Park). 2005;19(12):1634-938.

22. Stadler R, Greorcyk S, Euhus D, Place R, Huber P, Simmang C. Outcome of HIV-infected patients with invasive squamous-cell carcinoma of the anal canal in the era of highly active antiretroviral therapy. Dis Colon Rectum. 2004;47:1305-9. doi: https://doi.org/10.1007/s10350004-0584-1.

23. Wang C, Sparano J, Palefsky J. Human immunodeficiency virus/AIDS, human papillomavirus, and anal cancer. Surg Oncol Clin N Am. 2017;26:17-31. doi: https://doi. org/10.1016/j.soc.2016.07.010.

24. Ishida Y,Agata Y,Shibahara K,Honjo T. Induced expression of PD-1, a novel member of the immunoglobulin gene superfamily, upon programmed cell death. EMBO J. 1992;11:388795. doi: https://doi.org/10.1002/j.1460-2075.1992. tb05481.x.

25. Leach D, Krummel M, Allison J. Enhancement of antitumor immunity by CTLA-4 blockade. Science. 1996;271:1734-6. doi: https://doi.org/10.1126/science.271.5256.1734.

26. Ott P, Piha-Paul S, Munster P, Pishvaian M, van Brummelen E, Cohen R, et al. Safety and antitumor activity of the antiPD-1 antibody pembrolizumab in patients with recurrent carcinoma of the anal canal. Ann Oncol. 2017;28(5):103641. doi: https://doi.org/10.1093/annonc/mdx029.

27. Morris V, Salem M, Nimeiri H, Igbal S, Singh P, Ciombor $\mathrm{K}$, et al. Nivolumab for previously treated unresectable metastatic anal cancer (NCI9673): a multicenter, singlearm, phase 2 study. Lancet Oncol. 2017;18(4):446-53. doi: https://doi.org/10.1016/S1470-2045(17)30104-3.

28. Kim D, Byer J, Kothari N, Mahipal A, Chang Y, Kim R. EGFR inhibitors in patients with advanced squamous cell anal carcinomas: a single-institution experience. Oncology. 2017;92(4):190-6. doi: https://doi. org/10.1159/000452766.

29. Casadei A, Passardi A, Fornaro L, Rosetti P, Valgiusti M, Ruscelli S, et al. Treatment of squamous cell carcinoma of the anal canal: A new strategies with anti-EGFR therapy and immunotherapy. Crit Rev Oncol Hematol. 2018;123:52-6. doi: https://doi.org/10.1016/j.critrevonc.2018.01.007. 